\title{
Chapter 10 \\ The Future of Particle Physics: The LHC and Beyond
}

\author{
Ken Peach
}

\subsection{Update}

I have been asked to submit a revised version of this chapter, published almost a decade ago. However, I think that it is better to leave the historical record as it was - this was an article written in its time and for its time. If I was writing this article today, I would call it "The Future of Particle Physics-Beyond the LHC", in recognition of the fact that, when originally written, the LHC was still under construction and now it has been operating for several years. The other key event which informed the original article was the recently-developed European Strategy for Particle Physics, adopted by the CERN Council in July 2006; the strategy was updated in 2013 and formally adopted in May of that year [1]; as I write, the process of updating the strategy is under way.

The intervening decade has seen significant progress, most spectacularly in the observation of the Higgs scalar [3] at $125.10 \pm 0.14 \mathrm{GeV} / \mathrm{c}^{2}$ [2] which completes the Standard Model of the particles and their interactions (excluding gravity) which dominate the local region of the universe. This means that all of the free parameters of the Standard Model are now known with reasonable precision, which can be chosen as (see Table 10.1):

1. The quark masses $\left(m_{u}, m_{d}\right),\left(m_{c}, m_{s}\right),\left(m_{t}, m_{b}\right)$;

2. the charged lepton masses $m_{e}, m_{\mu}, m_{\tau}$;

3. the $Z^{0}$ mass $M_{Z}$;

4. the Higgs Mass $\mathrm{M}_{\mathrm{H}}$;

5. the electromagnetic coupling constant $\alpha$;

6. the strong coupling constant $\alpha_{\mathrm{s}}\left(\mathrm{M}_{\mathrm{Z}}\right)$;

K. Peach $(\square)$

John Adams Institute for Accelerator Science, Oxford, UK

e-mail: ken.peach@adams-institute.ac.uk 
Table 10.1 Parameters of the Standard Model [2]

\begin{tabular}{|c|c|c|c|}
\hline $\begin{array}{l}m_{u} \\
2.14_{-0.26}^{+0.49} \mathrm{MeV} / \mathrm{c}^{2}\end{array}$ & $\begin{array}{l}m_{c} \\
1.27 \pm 0.02 \mathrm{GeV} / \mathrm{c}^{2}\end{array}$ & $\begin{array}{l}m_{t} \\
172.9 \pm 0.4 \mathrm{GeV} / \mathrm{c}^{2}\end{array}$ & \\
\hline $\begin{array}{l}m_{d} \\
4.67_{-0.17}^{+0.48} \mathrm{MeV} / \mathrm{c}^{2}\end{array}$ & $\begin{array}{l}m_{s} \\
93_{-5}^{+11} \mathrm{MeV} / \mathrm{c}^{2}\end{array}$ & $\begin{array}{l}m_{b} \\
4.18_{-0.02}^{+0.03} \mathrm{GeV} / \mathrm{c}^{2}\end{array}$ & \\
\hline $\begin{array}{l}m_{e} \\
0.5109989461(31) \\
\mathrm{MeV} / \mathrm{c}^{2}\end{array}$ & $\begin{array}{l}m_{\mu} \\
105.6583745(24) \\
\mathrm{MeV} / \mathrm{c}^{2}\end{array}$ & $\begin{array}{l}m_{\tau} \\
1776.86(0.12) \mathrm{MeV} / \mathrm{c}^{2}\end{array}$ & \\
\hline $\begin{array}{l}\mathrm{M}_{\mathrm{Z}} \\
91.1876 \pm 0.0021 \\
\mathrm{GeV} / \mathrm{c}^{2}\end{array}$ & $\begin{array}{l}\mathrm{M}_{\mathrm{H}} \\
125.10 \pm 0.14 \mathrm{GeV} / \mathrm{c}^{2}\end{array}$ & & \\
\hline $\begin{array}{l}\alpha \\
7.2973525664(17) \\
\times 10^{-3} \\
\end{array}$ & $\begin{array}{l}\mathrm{G}_{\mathrm{F}} \\
1.1663787(6) \times 10^{-5} \\
\mathrm{GeV}^{-2}\end{array}$ & $\begin{array}{l}\alpha_{\mathrm{s}}\left(\mathrm{M}_{\mathrm{Z}}\right) \\
0.1179(10)\end{array}$ & \\
\hline $\begin{array}{l}\lambda \\
0.22453 \pm 0.00044\end{array}$ & $\begin{array}{l}\text { A } \\
0.836 \pm 0.015\end{array}$ & $\begin{array}{l}\rho \\
0.122 \pm 0.0175\end{array}$ & $\begin{array}{l}\eta \\
0.355 \pm 0.0115\end{array}$ \\
\hline Derived quantities & $\begin{array}{l}\sin ^{2} \theta_{W} \\
0.23129 \pm 0.00005\end{array}$ & $\begin{array}{l}M_{W}=M_{z} \cos \theta_{w} \\
80.385 \pm 0.015 \mathrm{GeV} / \mathrm{c}^{2}\end{array}$ & $\sin ^{2} 2 \theta_{w}=\frac{\alpha \pi}{8 G_{F} M_{Z}^{2}}$ \\
\hline
\end{tabular}

7. the weak coupling constant $\mathrm{G}_{\mathrm{F}}$; and

8. the Cabibbo-Kobayashi-Maskawa (CKM) parameters in the Wolfenstein parametrization $\lambda, A, \rho$ and $\eta$.

In addition, two other useful quantities are given- $\mathrm{M}_{\mathrm{W}}$ and $\sin ^{2} \theta_{\mathrm{W}}-$ which can be written in terms of the other parameters, as shown in the last line of Table 10.1.

This is a total of 18 parameters which can only (so far) be determined by experiment. However, once determined, they describe the observed interactions with remarkable precision; for example, the muon anomalous magnetic moment (averaged between $\mu+$ and $\mu-)$ is $\left(11,659,2091 \pm 54_{\text {stat }} \pm 33_{\text {syst }}\right) \times 10^{-11}$, to be compared with the theoretical expectation of $\left(11,659,1803 \pm 1_{\mathrm{EW}} \pm 49_{\mathrm{Had}}\right) \times 10^{-11}$, where the first error in the theoretical calculation comes from electroweak corrections and the second comes from a combination of low-order (42) and higher order (26) hadronic corrections [4]. The difference between these is $(288 \pm 80) \times 10^{-11}$ (3.5 standard deviations) and it is, of course, hotly debated whether this is statistically significant and, if it is, what that significance might be. There is a new experiment at Fermilab [5] which aims to reduce the total error in the muon anomalous magnetic moment to $14 \times 10^{-11}$, which, even without improvements to the theoretical calculations, would (if the central value of the discrepancy remained unchanged) increase the significance to more than 5 standard deviations.

Within the Standard Model, because the neutrinos have only one helicity state, they must be strictly massless. However, the phenomenon of neutrino oscillations, now well established, requires the neutrinos to have mass-essentially, the oscillation is a beating in the propagation through time of the different mass eigenstates that form the flavour eigenstates. The past decade has seen enormous progress in 
Table 10.2 Neutrino Oscillation Parameters $[6,7]^{\mathrm{a}}$

\begin{tabular}{|c|c|c|}
\hline & Normal hierarchy & Inverted hierarchy \\
\hline$\Delta m_{12}^{2}$ & \multicolumn{2}{|l|}{$\left|\Delta m_{23}^{2}\right|$} \\
\hline $7.37_{-0.14}^{+0.20} \times 10^{-5} \mathrm{eV}^{2} / \mathrm{c}^{4}$ & $2.50 \pm 0.04 \times 10^{-3} \mathrm{eV}^{2} / \mathrm{c}^{4}$ & $2.46 \pm 0.04 \times 10^{-3} \mathrm{eV}^{2} / \mathrm{c}^{4}$ \\
\hline $\operatorname{Sin}^{2} \theta_{12}$ & \multicolumn{2}{|l|}{$\operatorname{Sin}^{2} \theta_{23}$} \\
\hline $0.297_{-0.016}^{+0.019}$ & $0.439_{-0.019}^{+0.060}$ & $0.569_{-0.060}^{+0.023}$ \\
\hline $\operatorname{Sign}\left(\Delta m_{23}^{2}\right)$ & \multicolumn{2}{|l|}{$\operatorname{Sin}^{2} \theta_{13}$} \\
\hline$?$ & $0.0214_{-0.0010}^{+0.0011}$ & $0.0218_{-0.0011}^{+0.0010}$ \\
\hline$\Sigma \mathrm{m}_{v}$ & \multicolumn{2}{|l|}{$\delta / \pi$} \\
\hline$<0.183 \mathrm{eV}(95 \% \mathrm{CL})$ & $1.35_{-0.14}^{+0.21}$ & $1.32_{-0.22}^{+0.32}$ \\
\hline
\end{tabular}

${ }^{\text {a }}$ The 3 -sigma limits have been converted into 1-sigma asymmetric errors

the determination of the neutrino oscillation parameters (see Table 10.2). Although the mathematics of the flavour mixing is similar in the quark and neutrino sectors, the phenomenology is very different. In the quark sector, the flavour oscillationsdescribed by the CKM matrix - take place in either mixing or decay of bound states. In the neutrino sector-described by the Pontecorvo-Maki-Nakagawa-Sakata (PMNS) matrix - the flavour oscillations take place in vacuum, although there is an additional term for neutrino transmission in matter to take account of the different interaction cross-sections of the electron neutrinos, and the muon and tau neutrinos (and, of course, their antineutrinos). The main uncertainty is whether the third neutrino mass is heavier than the other two (normal hierarchy) or lighter (inverted hierarchy), with an emerging preference [8] for normal hierarchy. Most impressive is the evidence that the CP-violating phase angle in the PMNS matrix ( $\delta$ ) is non-zero $-0 / 2 \pi$ is excluded at the 3 -standard deviation level, and $\pi$ at 2.5 (1.5) standard deviations for the normal (inverted) hierarchy. The best limits on the absolute neutrino mass scale come from cosmology, giving an upper limit on $\Sigma \mathrm{m}_{\nu}$ of $0.183 \mathrm{eV}$ at the $95 \%$ confidence level [7]. There are essentially two ideas for extending the Standard Model to include finite neutrino masses. The first notes that, since the neutrino has no electric charge, it is possible to construct a masslike term from the left-handed neutrino and right-handed-antineutrino fields, which changes its nature to that of a Majorana particle [9]. (If the neutrinos are indeed Majorana particles, there are two additional phase angles ( $\alpha$ and $\beta$ ) in the PMNS matrix which are, however, extremely difficult to measure.) The second postulates the existence of a heavy right-handed neutrino which, in combination with a very high mass scale (like the GUT scale), produces through the "see-saw" mechanism one light left-handed neutrino $\left(<<1 \mathrm{eV} / \mathrm{c}^{2}\right)$ and a heavy right-handed neutrino $\left(>>10^{12} \mathrm{GeV} / \mathrm{c}^{2}\right.$ ) - as the left-handed neutrino becomes lighter the right-handed neutrino becomes heavier through the "see-saw". One consequence of these is that there are interactions that allow a change of lepton number by 2 units, thus enabling neutrinoless double- $\beta$ decay to occur. (For a recent review, see [10].)

The other area where the Standard Model obviously fails is in the Dark SectorDark Matter and Dark Energy. The cosmological and astronomical evidence for 
these is overwhelming (see reference [11] for a recent review) with a recipe for the current state of the Universe containing about 70\% of Dark Energy, 25\% Dark Matter and 5\% "normal" matter, the only part addressed by the Standard Model. From a particle physics perspective, Dark Matter is more tractable. First, there is the challenge of directly detecting it using large volume, sensitive detectors deep underground. The past decade has seen the limits steadily improved (see reference [12] for a recent review), while the small number of claimed signals have still not been independently confirmed. There is an ongoing programme of experiments at the major underground laboratories which should improve these limits by several order of magnitude over the next few years. Second, there are straightforward extensions of the Standard Model which provide candidates for Dark Matter. Unfortunately, there is no reliable estimate for the mass scale at which these new particles might appear. Such evidence might come from the Dark Matter searches themselves. The other place where a mass scale might be forthcoming is in the analysis of the deficiencies of the Standard Model. For example, Supersymmetry was proposed to address some hierarchy problems within the model, and also to assist in Grand Unification, both of which pointed to a $\mathrm{TeV}$ energy scale for appearance of new particles. However, so far these have failed to materialize at the LHC; unfortunately, the parameter space for Supersymmetry is extensive and so, while this might be disappointing it may not be conclusive. Dark Energy, on the other hand, is still mysterious and it is unclear where it fits in to the global picture; its understanding might require the reconciliation of quantum mechanics with general relativity.

The "open questions within the Standard Model" identified in the original article have been largely resolved, although there are still details to be addressed, for example the transition from perturbative to non-perturbative QCD. However, the "open questions beyond the Standard Model" remain, refined somewhat; for example, the "pattern of fermion masses" might nowadays be cast as "understanding the Yukawa couplings of the fundamental fermions to the Higgs". The improvements in the knowledge of the neutrino oscillation parameters do not explain how the mixing comes about, nor how the neutrinos derive their (tiny) masses. Again, there has been much progress in the measurement of the various cosmological parameters, including those which either determine or are determined by particle physics, but the "open questions in particle physics and cosmology" are still far from resolved. For example, the arguments for Grand Unification of the strong and electroweak forces remain intact (see Fig. 10.1) even though there is, as yet, no direct evidence for the existence of Supersymmetric particles. A second area which points to new physics is to note that, of the 18 parameters that define the Standard Model, 11 are directly related to mass (the quarks, the charged leptons, the $\mathrm{Z}$ and the Higgs), which becomes 12 if the Fermi constant is replaced (as it can be) by the mass of the W. Given this, there are three mass hierarchies to be explained. First, within a generation, what determines the relative masses of the charged lepton, the downtype quark (charge -1/3) and the up-type quark (charge $+2 / 3$ ) (and why is the up-quark lighter than the down quark, while the charm and top quarks are heavier than the strange and bottom quarks)? Second, what determines the large (several 

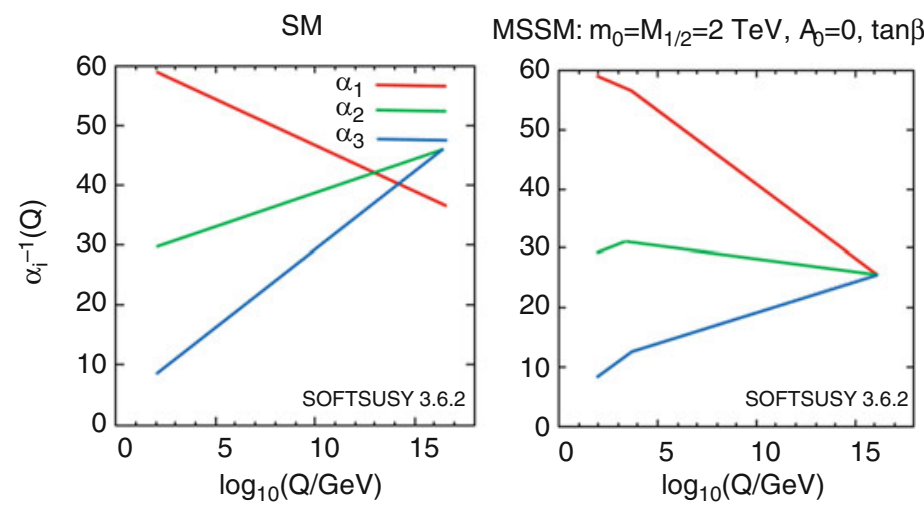

Fig. 10.1 The evolution of the fundamental forces as a function of energy in the standard model (left) and a particular set of parameters in the Minimal Supersymmetric Standard Model (right). (Adapted from [13])

orders of magnitude) mass differences between the generations of the same type of fundamental fermion. Third, what determines the masses of the heavy field bosons (Higgs and the $\mathrm{W}$ and $\mathrm{Z}$ )? (The other gauge bosons - the photon and the gluonremain massless, protected by an unbroken gauge symmetry.) The origin of the mixing matrix and CP-violation in the quark sector is still unresolved, as are the masses and oscillation parameters in the neutrino sector.

The detection of Gravitational Waves from the merger of two black holes [14] and the subsequent detection of gravitational waves from a binary neutron star inspiral [15] open the door to a new era of observational astronomy of matter under extreme conditions, and may also provide insight into the long-standing issue of the incompatibility of general relativity with quantum mechanics. Resolving this is likely to require understanding of physics at the Planck scale.

Given this, the main conclusions of the "Way Forward" are largely unchanged. Clues to the physics that must exist beyond the Standard Model could come directly from the observation of new states of matter (e.g. Supersymmetry), either at the LHC or at a new energy frontier machine, or indirectly from precision measurements of Standard Model parameters, for example at a Linear Collider built to produce large numbers of Higgs particles under clean conditions. Alternatively, ultrahigh precision measurements of low energy parameters ( $\mathrm{g}-2, \mu \rightarrow$ e conversion, neutrinoless double $\beta$ decay ...) as well as new astrophysical observations (Dark Matter, gravitational waves) could give strong pointers toward the new physics. The tremendous progress in the neutrino sector from, particularly, T2K and Nova probably means that the arguments in favour of a neutrino factory have weakened somewhat, but the arguments for the other facilities discussed (including the luminosity upgrade for the LHC) remain and have strengthened.

A recent publication by the T2K collaboration (Nature 580, 339-344 (2020)) reports that "The $3 \sigma$ confidence interval for $\delta_{\mathrm{CP}}$, which is cyclic and repeats every 
$2 \varphi$, is $[-3.41,-0.03]$ for the so-called normal mass ordering and $[-2.54,-0.32]$ for the inverted mass ordering".

\section{References}

1. The European Strategy for Particle Physics, http://cds.cern.ch/record/1567258/files/esc-e106.pdf?subformat=pdfa

2. Patrignani, C et al. (Particle Data Group), Chin. Phys. C 40, 100001 (2016) and 2017 update; M. Tanabashi et al. (Particle Data Group), Phys. Rev. D 98, 030001 (2018) and 2019 update

3. Aad, G et al. (ATLAS Collaboration) Phys. Lett. B 716 1-29 (2012); Chatrchyan, S et al. (CMS Collaboration) Phys. Lett. B 716 30-61 (2012)

4. Hoecker, A and Marchiano, WJ, in J. Beringer et al. (Particle Data Group), Phys. Rev. D 86, 010001 (2012), updated in 2013

5. The Muon g-2 collaboration, co-spokespersons Roberts, BL and Hertzog, DW, http://muon-g2.fnal.gov/

6. Nakamura, K, Petcov, ST in Patrignani, C et al. (Particle Data Group), Chin. Phys. C 40, 100001 (2016)

7. Giusarma, E et al., Phys. Rev. D 94, 083522 (2016); a recent analysis by Couchot F at al., to be published in Astronomy and Astrophysics, reports an upper limit of $0.17 \mathrm{eV}$.

8. Adamson, P et al, Phys. Rev. Lett. 118, 231801 (2017); Abe,K arXiv:1707.01048 [hep-ex] (2017)

9. Majorana, E, Il Nuovo Cimento 14 171-184 (1937)

10. Dell'Oro, S, Marcocci, S, Viel, M and Vissani, F, Advances in High Energy Physics 2016 2162659 (2016)

11. Arun, K, Gudennavar, SB and Sivaram, C, Advances in Space Research 60 166-186 (2017)

12. Liu, J, Chen, X and Ji, X, Nature Physics 13 212-216 (2017)

13. Hebecker, A and Hisano, J in in Patrignani, C et al. (Particle Data Group), Chin. Phys. C 40, 100001 (2016)

14. Abbott BP et al. (LIGO Scientific Collaboration and VIRGO Collaboration), Phys. Rev. Lett. 116061102 (2016)

15. Abbott BP et al. (LIGO Scientific Collaboration and VIRGO Collaboration), Phys. Rev. Lett. 119161101 (2017)

Open Access This chapter is licensed under the terms of the Creative Commons Attribution 4.0 International License (http://creativecommons.org/licenses/by/4.0/), which permits use, sharing, adaptation, distribution and reproduction in any medium or format, as long as you give appropriate credit to the original author(s) and the source, provide a link to the Creative Commons licence and indicate if changes were made.

The images or other third party material in this chapter are included in the chapter's Creative Commons licence, unless indicated otherwise in a credit line to the material. If material is not included in the chapter's Creative Commons licence and your intended use is not permitted by statutory regulation or exceeds the permitted use, you will need to obtain permission directly from the copyright holder. 